\title{
Conocimientos y percepción sobre el tabaquismo y sus efectos dañinos a la salud entre los estudiantes adolescentes de Venecia y Aguas Zarcas, Costa Rica
}

\author{
Nick Bohrer \\ St. Olaf College, 1500 St. Olaf Ave, Northfield, MN 55057, (715) 523-0365; nickbohrer12@gmail.com
}

Recibido 9-X-2013 • Corregido 28-II-2014 • Aceptado 4-III-2014

\begin{abstract}
Tobacco addiction represents one of the most grave worldwide public health problems, especially within populations of developing countries. Studies in Costa Rica and other countries demonstrate that this addiction frequently begins during adolescence. This study evaluated with a written questionnaire the general and specific knowledge of 529 students between the ages of 12 and 18 years of the health risks associated with tobacco addiction. It also evaluated their perceptions of the factors that predict the onset of adolescent tobacco addiction. The questionnaire was applied in three of the schools of Venecia and Aguas Zarcas, districts of the San Carlos canton of Costa Rica. The students demonstrated a basic knowledge of the health risks of tobacco addiction on the lungs, pregnancy complications, and cancer. The study indicated a lack of student knowledge of the adverse effects of tobacco on cardiovascular and female reproductive health. There existed a positive linear relation between median knowledge level and age $\left(P \leq 0,0001 ; R^{2}=5,45\right)$, as well as academic grade $\left(P \leq 0,0001 ; R^{2}=10,2\right)$. The predictive factors of adolescent tobacco addiction considered the most influential by the students were the influence of smokers within family and friend groups. The implementation of a comprehensive academic program for prevention of tobacco use and addiction that addresses both lack of knowledge and perceptions of these students related to tobacco addiction is recommended.
\end{abstract}

Key words: tobacco addiction, smoking, health risks, adolescents, perceptions, San Carlos (Costa Rica).
RESUMEN: El tabaquismo representa uno de los problemas de salud pública más frecuentes a nivel mundial, y afecta especialmente a las poblaciones de países en desarrollo. Estudios de Costa Rica y otros países evidencian que esta adicción frecuentemente se inicia durante la adolescencia. Esta investigación pretendió evaluar, mediante un cuestionario escrito, conocimientos generales y específicos de 529 estudiantes de edades entre 12 y 18 años de los efectos adversos del tabaquismo a la salud. También evaluó su percepción sobre los factores que llevan al tabaquismo en adolescentes. Se aplicó el cuestionario en tres de los colegios de Venecia y Aguas Zarcas, distritos del cantón de San Carlos, Costa Rica. Los estudiantes comunicaron un reconocimiento básico de los daños del tabaquismo en los pulmones, las complicaciones al embarazo, y el cáncer. La investigación no evidenció un conocimiento por la parte de los estudiantes de los efectos adversos que tiene el tabaco en la salud cardiovascular y en la reproductiva femenina. Había una relación linear positiva entre la media nivel de conocimientos y edad $\left(P \leq 0,0001 ; R^{2}=5,45\right)$, además de grado académico $\left(P \leq 0,0001 ; R^{2}=10,2\right)$. La percepción más frecuente de los factores que llevan al tabaquismo en adolescentes tenía que ver con las influencias de los familiares y amigos fumadores. Se recomienda la implementación de un programa académico de prevención que responda a la falta de conocimientos y a la percepción de estos estudiantes acerca del tabaquismo.

Palabras claves: consumo de tabaco, fumar, daños al cuerpo, jóvenes, factores de riesgo, San Carlos (Costa Rica).
El tabaco es una planta que se utiliza para fabricar el cigarrillo y otros productos de consumo. Estos productos además contienen la nicotina, agente adictivo, y otras sustancias potencialmente dañinas a la salud, como el alquitrán, el monóxido de carbono, y muchos agentes cancerígenos (Centers for Disease Control and Prevention, 2013). Se estima que el humo del cigarrillo contiene más de 5000 sustancias, de los cuales 98 son conocidas por potencialmente aumentar el riesgo de cáncer, enfermedades cardiovasculares y respiratorias, y otras complicaciones en el fumador (Talhout et al., 2011).

Los daños en el sistema respiratorio asociados con el consumo de tabaco incluyen el cáncer de pulmón, bronquitis, neumonía, enfisema y retardo del crecimiento de los pulmones de adolescentes (Surgeon General's Report, 2004). El cáncer de pulmón causa un $29 \%$ y $26 \%$ de los casos mundiales de cáncer para el hombre y la mujer, respectivamente (Steliga \& Dresler, 2011). 
Los daños al sistema reproductivo femenino causados por el consumo de tabaco incluyen el aumento del riesgo de la concepción tarde y la infertilidad (Surgeon General's Report, 2004). En la madre tabaquista ocurren un $5-10 \%$ de los muertes perinatales, el $20-35 \%$ de los nacimientos de peso bajo y el 8-15\% de los partos prematuros (Clark \& Nakad, 2011). El fumar de la embarazada también se ha asociado con daños en el desarrollo del feto que llevan a la función reducida del pulmón, discapacidades cognitivas y otros problemas (Abbott \& Winzer-Serhan, 2012).

En cuanto al sistema cardiovascular, se encontró del uso regular de tabaco sin humo una relación con la aterosclerosis (Rahman, Mahmood, Spurrier, Rahman \& Leeder, 2011), y con el fumar el mismo (Prasad, Kabir, Das \& Dash, 2009) además de la aneurisma de aorta y el derrame cerebral (Surgeon General's Report, 2004).

El fumar puede llevar a varios tipos de cáncer aparte del pulmón, incluyendo el cáncer de colón, cérvix, vejiga, esófago, riñón, laringe, boca, páncreas y estómago, y la leucemia (Surgeon General's Report, 2004). Además, el tabaquismo debilita el sistema inmunológico y predispone el cuerpo a varias infecciones bacteriales (Feldman \& Anderson, 2013), y aumenta el riesgo de adquirir una catarata, fractura de cadera, densidad baja de los huesos, úlcera péptica, y una disminución general de la salud (Surgeon General's Report, 2004).

Según un estudio nacional del Instituto sobre Alcoholismo y Farmacodependencia (IAFA) de 2012, el $24,8 \%$ de la población costarricense entre los 12 y 70 años había consumido el tabaco. El $13,4 \%$ de la población lo había consumido durante el mes antes del estudio (IAFA, 2012). Otro estudio encontró que el $28,6 \%$ de los adolescentes costarricenses de nivel de escolaridad $7^{\circ}$ a $11^{\circ}$ ya había consumido el tabaco, y el $8,9 \%$ durante el mes antes del estudio. La edad media del inicio de fumar era de 12,6 años (IAFA, 2011).

Cuando la Organización Mundial de la Salud evaluó la frecuencia del tabaquismo entre estudiantes de 13-15 años de edad, Costa Rica representó la quinta más alta $(44,4 \%)$ de dieciséis locaciones internacionales encuestadas, en el número de participantes que había probado un cigarrillo. Solo el $37,8 \%$ de los estudiantes costarricenses afirmó que había aprendido, en su colegio durante el año antes del estudio, de los efectos de fumar, el número cuarto más bajo de las dieciséis locaciones. Sin embargo, todavía falta un análisis de cuáles daños a la salud del tabaquismo conoce esta población, o una similar (Warren et al., 2000).
En 2012, la Asamblea Legislativa de La República de Costa Rica aprobó la Ley general del control de tabaco y sus efectos nocivos a la salud (Gobierno de Costa Rica, 2012). Esta ley intenta disminuir el tabaquismo en Costa Rica mediante medidas variadas que incluye el establecimiento del Programa Nacional de Educación para la Prevención e Información sobre el Consumo del Tabaco, un esfuerzo coordinado entre el Ministerio de Salud y el Ministerio de la Educación Pública. Sin embargo, la ley no incluye la implementación de un plan para el programa educativo.

Tanto las áreas de conocimiento como la percepción de los adolescentes del tabaquismo influyen en sus decisiones acerca del consumo de tabaco. Varios estudios han encontrado una relación entre la frecuencia del tabaquismo adolescente y la percepción del nivel de riesgo asociado con el fumar, además del ambiente social y familiar del adolescente (Nebot et al., 2004; Song et al., 2009). Entre estos, existe evidencia de la importancia de estos factores específicamente para adolescentes latinoamericanos (Bejarano et al., 2011; Bolzan \& Peleteiro, 2003).

Los objetivos de este estudio son: a) determinar el nivel de conocimiento de los estudiantes adolescentes entre las edades de 12 y 18 años de Venecia y Aguas Zarcas sobre los daños del tabaquismo a la salud; b) identificar los factores que influyen en adquirir el hábito de fumar en estos adolescentes; c) identificar si existe relación entre el nivel de conocimiento sobre los daños del tabaquismo según la edad, el sexo y el colegio de los participantes.

\section{METODOLOGÍA}

Región y Muestra de Estudio: El estudio se realizó en San Carlos, el cantón número diez de la provincia de Alajuela, Costa Rica, en los distritos de Venecia y Aguas Zarcas, pueblos rurales con poblaciones estimadas de 7 394 y 13 651, respectivamente (INEC, 2011).

La muestra del estudio consistió en 529 estudiantes en total, de 44 grupos de estudiantes cuyas horas guías concidieron con las visitas del investigador a sus colegios. Se incluyeron estudiantes con edades entre 12 y 18 años, y grados de 7 a 12, de tres colegios en los distritos de Venecia y Aguas Zarcas: el Colegio Técnico Profesional de Venecia, el CINDEA (Centros Integrados de Educación para Jóvenes y Adultos) de Venecia, y el Colegio Técnico Profesional de Aguas Zarcas. Los dos colegios técnicos profesionales cuentan con estudiantes divididos entre los grados de 7-12, con un mínimo de 12 años de edad. EI CINDEA, un colegio nocturno, consiste en estudiantes 
con un mínimo de 15 años de edad, y no divididos en grados específicos.

El Cuestionario y su Aplicación: Se evaluaron los conocimientos y percepción de los adolescentes de San Carlos sobre el tabaquismo y sus daños a la salud por medio de un cuestionario (Apéndice A). Este fue diseñado por el investigador y revisado por varios asesores, incluyendo la Dra. Anabelle Alfaro Obando. Además un estudiante de San José de quince años rellenó el cuestionario, para asegurar que las preguntas fueran comprensibles (sus respuestas no fueron incluidas en el análisis). La primera parte comenzó con una pregunta para anotar todas las enfermedades y complicaciones del tabaquismo de que sabe el participante. Posteriormente se evaluó, de acuerdo a la percepción del estudiante, los factores que pueden influir para adquirir el hábito del tabaquismo, y se le dio al estudiante la opción de apuntar otro que el considerara importante.

Las preguntas de la segunda parte pretendían que el encuestado explicara con más detalle sus conocimientos sobre los daños del tabaco en ciertos sistemas específicos: los pulmones, el corazón y las arterias, y el embarazo y la salud reproductiva femenina. Para evitar que las preguntas de la segunda parte influyeran las respuestas a la primera, se entregaron en forma separada.

Se aplicó la encuesta con ciertos grupos de estudiantes durante su hora guía. Para los estudiantes del grado 12 y los del CINDEA de Venecia que no tenían una hora guía, se dio la encuesta durante una lección con permiso del profesor. Todos los estudiantes de los grupos seleccionados tuvieron la opción de participar en el estudio voluntariamente. Se consiguieron las firmas de consentimiento informado de todos los participantes, además de los padres de los estudiantes menores de edad. Entonces se distribuyó el cuestionario entre los participantes, y se dio 40min para terminar ambas partes del cuestionario.

Se categorizaron las respuestas de las preguntas sobre los daños del tabaco a la salud entre tres niveles, Nivel 1, Nivel 2, y Nivel 3, basados en el número de daños ciertos mencionados en la respuesta (Tabla 1). Se compiló una lista de estos efectos conocidos con certeza a la salud asociados con el tabaquismo, basados en el informe The Health Consequences of Smoking: a Report of the Surgeon General (Surgeon General's Report, 2004), además de otros estudios que sugieren otros efectos adversos. También con la pregunta sobre los daños generales, la pregunta sobre los factores que pueden predisponer al adolescente al tabaquismo, y la pregunta sobre el daño más grave, se sumó cuantas veces se escribió cada una de las respuestas más frecuentes.

Análisis de Datos: Se evaluaron los niveles de las respuestas a cada pregunta contra los factores de edad, nivel de escolaridad, sexo y colegio, mediante la aplicación de pruebas estadísticas de los programas Microsoft Excel y Statgraphics Centurion XVI (Statpoint Technologies, Inc., www.statgraphics.com/statpoint.htm). Se hizo análisis de regresión univariada entre las respuestas y la edad además del grado de los estudiantes. Además, se hicieron pruebas paramétricas univariadas de ANOVA entre las respuestas y la edad, el grado, el sexo y el colegio, para buscar medias significativamente diferentes.

\section{RESULTADOS}

Un total de 529 estudiantes, 311 mujeres (58,8\%) y 218 hombres (42,2\%), de los tres colegios participaron en el

TABLA 1

Descripciones de las respuestas de Nivel 1, Nivel 2 y Nivel 3, de las preguntas sobre los conocimientos de los daños del tabaquismo a la salud.

Para la pregunta sobre los daños generales, la pregunta sobre los efectos en los pulmones, y la pregunta sobre los efectos en el embarazo y la salud reproductiva femenina:

Nivel 1

La respuesta no incluyó ningún efecto cierto del tabaquismo a la salud.

Nivel 2

Nivel 3

La respuesta incluyó uno (1) o dos (2) efectos ciertos del tabaquismo a la salud.

Para la pregunta sobre los efectos en el corazón y las arterías*

Nivel 1

La respuesta no incluyó ningún efecto cierto del tabaquismo a la salud.

Nivel 2

La respuesta incluyó un (1) efecto cierto del tabaquismo a la salud.

Nivel 3

La respuesta incluyó dos (2) o más efectos ciertos del tabaquismo a la salud.

\footnotetext{
* Se evaluó esta pregunta de modo diferente a causa de la cantidad menor de esta clase de efectos conocidos con certeza.
} 
estudio. La muestra incluyó estudiantes de cada año de edad entre 12 y 18 y cada grado entre 7 y 12 . La edad media fue de 15,2 años.

El 98,3\% de los participantes demostró el conocimiento de por lo menos un efecto adverso del tabaquismo a la salud. La respuesta media de nivel de esta pregunta fue 2,37, más alta que la de las preguntas de daños en sistemas específicos ( $F=172 ; d f=3,2112 ; P \leq 0,0001)$. Se obtuvo una relación lineal, positiva fuerte de respuesta a mayor edad $\left(F=30,4 ; d f=1,527 ; P \leq 0,0001 ; R^{2}=5,45\right)$ y a mayor grado o año de colegio cursado $(F=56 ; d f=1,493$; $\left.P \leq 0,0001 ; R^{2}=10,2\right)$. La respuesta media de nivel de alumnos de grado 7 fue más baja $(\bar{X}=2.14)$ que la de alumnos del grado $12(\bar{X}=2.56)(F=12,44, d f=5,489 ; P \leq 0,0001)$. Sin embargo, no había una relación entre la respuesta media de nivel con el sexo (género), ni el colegio.

Los efectos del tabaquismo a la salud más frecuentemente mencionados fueron los relacionados con los pulmones/la respiración (456 veces, por $86,2 \%$ de los participantes) y los relacionados con alguna forma de cáncer (411 veces, por $77,7 \%$ de los participantes). También mencionados frecuentemente (más de 40 veces) fueron el cáncer de pulmón, asma/tos, daños a los dientes, problemas mentales, daños al sistema cardiovascular, cáncer de algún órgano aparte del pulmón, y pulmonía.

El 90,0\% de los participantes demostraron conocimiento de por lo menos un daño a los pulmones. La respuesta media de nivel fue 1,97, con una relación lineal, positiva entre la respuesta media de nivel y la edad $\left(F=9,51, d f=1,527 ; P=0,0021 ; R^{2}=1,77\right)$ además del grado escolar $\left(F=5,43, d f=1,493 ; P=0,0202 ; R^{2}=1,09\right)$. No había una relación entre la respuesta media de nivel y el sexo (género), ni el colegio.

El $65,7 \%$ de los participantes demostraron conocimiento de por lo menos un daño al corazón y las arterías. La respuesta media de nivel fue 1,72 , con una relación lineal, positiva entre la respuesta media de nivel y la edad $\left(F=8,57, d f=1,527 ; P=0,0036 ; R^{2}=1,60\right)$ además del grado escolar ( $\left.F=9,80, d f=1,493 ; P=0,0018 ; R^{2}=1,95\right)$. No había una relación entre la respuesta media de nivel y el sexo (género), ni el colegio.

El $89,8 \%$ de los participantes demostraron conocimiento de por lo menos un daño al embarazo y al sistema reproductivo femenino. La respuesta media de nivel fue 1,93. La respuesta media de nivel fue menor para los alumnos ( $\bar{X}=1,87)$ que para las alumnas $(\bar{X}=1,98)$ $(F=10,95, d f=1,527 ; P=0,0010)$ (Apéndice digital). No había una relación entre la respuesta media de nivel y la edad o el grado, ni el colegio.

Al analizar la percepción de los factores que predisponen al adolescente al tabaquismo, se encontró que los factores más influyentes para los participantes fueron él de tener amigos que usan productos de tabaco $(\bar{X}=2,38$, la media más alta) y él de tener padres o hermanos que fuman ( $\bar{X}=2,37$, la media segunda más alta).

El factor menos influyente fue él de pasar tiempo en fiestas, con una respuesta moda de nivel de influencia de 2 (para todos los otros factores la moda fue 3). No había una relación entre las respuestas medias o modas de nivel y el sexo (género), la edad o el grado.

De los 529 participantes, 133 escribieron otro factor que predispone al adolescente al tabaquismo, siendo los mas frecuentes el tener problemas familiares, la presión social, y la autoimagen/popularidad del tabaco.

De los 529 participantes, 482 respondieron acerca de su opinión del daño más grave del tabaquismo a la salud. Muchos participantes escogieron más de un daño. Las respuestas más comunes fueron los daños a los pulmones/la respiración, alguna forma de daño asociado con el cáncer, y la muerte.

\section{DISCUSIÓN}

Los estudiantes encuestados conocían que el tabaco puede llevar al cáncer y a daños a los pulmones, que se podría atribuir a la gran frecuencia de estos tipos de enfermedades en la sociedad, tanto al nivel mundial que al nacional (Steliga \& Dresler, 2011; WHO Costa Rica, 2011).

Al apuntar los daños a la salud en general del tabaco, la mayoría de los estudiantes excluyó los producidos en el embarazo. Sin embargo, casi el $90 \%$ pudo explicarlos en la pregunta sobre el embarazo. Esta discrepancia por lo menos sugiere que los estudiantes, a pesar de tener una comprensión básica de los efectos al embarazo, no los reconocieron como tan frecuentes como los a los pulmones y como causa del cáncer.

Aunque la mayoría de los estudiantes pudo mencionar los efectos adversos a los pulmones y el cáncer en general, no se demostró en la investigación una comprensión más detallada de los daños. Esto indica un conocimiento limitado de estos estudiantes del deterioro que causa el tabaco en varios sistemas del cuerpo, a corto y a largo plazo.

Se detectó un desconocimiento de los efectos adversos al sistema cardiovascular, en que solamente el 34\% de los estudiantes pudo mencionar un daño cierto. El $60 \%$ mencionó la relación del tabaquismo con la aterosclerosis y/o el peligro del paro cardiaco. Solamente dos estudiantes mencionaron un daño cardiovascular en el cerebro (i.e. el derrame, el aneurisma). 
Se reveló también un desconocimiento de los efectos del tabaco a la salud reproductiva femenina. Casi ningún estudiante mencionó el cáncer de cérvix o la infertilidad. Es posible que el desconocimiento del cáncer de cérvix como efecto adverso del tabaquismo proviene de una falta de comprensión de la enfermedad en general. Esto se evidenció en los resultados de un estudio en estos mismos colegios hace un año (Carlson, 2012). Un hallazgo similar a lo detectado en esta investigación fue lo también reportado de un estudio que encuestó a unas empleadas de un hospital de Connecticut (Roth \& Taylor, 2001). Se encontró que aún estas mujeres desconocían los daños de fumar a la salud femenina. Estos resultados confirman que la falta de reconocimiento de la amplitud y gran variedad de los daños del tabaquismo prevalece entre diferentes edades, culturas y niveles de educación.

Se encontró un aumento con edad y grado de lo que sabían los estudiantes de la amplitud de daños a la salud del tabaquismo. Sin embargo, aún entre los grupos de estudiantes con los niveles más altos de conocimiento, los de 17 años y los del duodécimo grado, la mayoría no pudo mencionar más de dos daños. Esta tendencia positiva de más conocimientos con más edad y con más años escolares por lo menos destaca el potencial para ampliar y profundizar estos conocimientos mediante el establecimiento de programas educativas de prevención.

De todos los factores predictivos posibles, los participantes opinaron que la familia y los amigos del adolescente tienen una influencia particular en su decisión de iniciar el consumo de tabaco. Esto también fue descrito por estudios en Barcelona y la Argentina que encontraron una correlación positiva entre estos factores y la tendencia de sus participantes, ambos hombres y mujeres, de iniciar el uso del tabaco (Bolzan \& Peleteiro, 2003; Nebot et al., 2004). El estudio Nebot en Barcelona encontró también una relación positiva entre la frecuencia del tabaquismo adolescente y el pasar tiempo en entornos de consumo frecuente de tabaco, alcohol y otras drogas. Los estudiantes de San Carlos, por otro lado, evaluaron el factor de pasar tiempo en fiestas (un entorno asociado con el consumo de tabaco, alcohol y otras drogas) como relativamente menos influyente que los otros. Sin embargo, cada uno de los factores apuntados en la encuesta fue evaluado por los participantes típicamente como por lo menos "un poco" influyente. Esto refleja su percepción que el tabaquismo adolescente es un problema que puede resultar de una variedad de factores ambientales y personales.

Se recomienda que se aproveche de los resultados de esta investigación, específicamente los elementos desconocidos y la percepción de los estudiantes con respeto al tabaco, para lograr disminuir la probabilidad de que el adolescente adquiera el hábito de consumir tabaco. Esto se realizaría por medio del establecimiento de programas educativos de prevención entre los colegios.

El desconocimiento de los efectos adversos a la salud cardiovascular que el tabaquismo produce merece atención, especialmente dado la frecuencia de muertes relacionadas con daños al sistema cardiovascular en las zonas rurales de Costa Rica (Campos et al., 1992; WHO Costa Rica, 2011). La relación que tiene el consumo del tabaco con la salud reproductiva femenina también se debe enfatizar en programas de prevención. Sería importante entrar en el tema del cáncer de cérvix, como una enfermedad muy frecuente entre la población femenina costarricense (Carlson, 2012). El reconocimiento del tabaquismo como uno de los factores que contribuyen a estas enfermedades podría ayudar a cambiar la percepción de los estudiantes menos convencidos de los peligros más conocidos del consumo de tabaco, como el cáncer de pulmón.

La otra faceta de un programa educativo de prevención abordaría los otros factores que más influyen la tendencia al tabaquismo adolescente: los hábitos de su familia y amigos con respeto al tabaco. Medidas que responden a estos factores podrían incluir la enseñanza de estrategias para superar la presión de los pares, además de programas que involucren a los padres del adolescente en apoyar el programa y reforzar sus mensajes en la casa.

La organización del gobierno federal estadounidense "Centers for Disease Control and Prevention" (Centros para el Control y Prevención de Enfermedades) ha publicado un informe detallado con directrices para implementar un programa institucional para prevenir el tabaquismo adolescente, que incluye muchas de las medidas mencionadas anteriormente (Centers for Disease Control and Prevention, 1994).

Limitaciones del Estudio: La aplicación de la encuesta en las clases de los dos colegios presentó varios obstáculos. Con los grupos que se pudieron encontrar el segundo día, más de la mitad de los estudiantes de cada grupo no entregó el formulario de consentimiento firmado por un padre o encargado. Durante la aplicación de la encuesta, fue difícil a veces prevenir que se compartiera información sobre las preguntas entre grupos pequeños de estudiantes.

La limitación más grande de esta investigación fue la incapacidad por razones de ética de preguntar a los estudiantes si ellos o algunos familiares consumen tabaco. Esa información habría sido muy útil para analizar la 
relación entre la frecuencia del hábito y los factores de edad, sexo y colegio, además de conocimiento y percepción de los daños a la salud del tabaco.

Investigaciones para el Futuro: A pesar del número de estudios previos que han analizado los factores que afectan la tendencia hacia el tabaquismo adolescente, parece haber una falta de estudios que busquen una relación específica entre el nivel de conocimiento de adolescentes de los daños a la salud del tabaquismo y la tendencia hacia la adicción.

Considero es importante realizar este mismo estudio a una población similar de estudiantes en un colegio de otro país como los EEUU. También sería pertinente realizar este estudio en un colegio costarricense con un programa académico de prevención ya establecido.

\section{AGRADECIMIENTOS}

El investigador expresa su más sincero agradecimiento a su asesora de la investigación Anabelle Alfaro Obando, y el profesorado de la ACM, especialmente a Chris Vaughan, Michael McCoy, Mario Morera y León Narváez de St. Olaf College. Muchas gracias a los directores y al cuerpo docente de los colegios de Venecia y Aguas Zarcas por su gran hospitalidad y apoyo, y, por supuesto, a los estudiantes por participar con tanto entusiasmo en el estudio.

\section{REFERENCIAS}

Abbott, L. C., \& Winzer-Serhan, U. H. (2012). Smoking during pregnancy: lessons learned from epidemiological studies and experimental studies using animal models. Critical Reviews in Toxicology, 42(4), 279-303. doi: 10.3109/10408444.2012.658506

Bejarano, J., Ahumada, G., Sanchez, G., Cadenas, N., de Marco, M., Hynes, M., \& Cumsille, F. (2011). Perception of risk and drug use: an exploratory analysis of explanatory factors in six Latin American countries. The Journal of International Drug, Alcohol and Tobacco Research, 1(1), 9-17.

Bloom, B., Adams, P. F., Cohen, R. A., \& Simile, C. (2012). Smoking and oral health in dentate adults aged 18-64. NCHS data brief, (85), 1-8.

Bolzan, A., \& Peleteiro, R. (2003). Smoking during early adolescence. A study in schoolchildren from Argentina. [Tabaquismo durante la adolescencia temprana. Estudio en escolares argentinos.]. Jornal de pediatria, 79(5), 461466. doi: 10.1590/s0021-75572003000500015
Campos, H., Mata, L., Siles, X., Vives, M., Ordovas, J. M., \& Schaefer, E. J. (1992). Prevalence of cardiovascular risk-factors in rural and urban Costa Rica. Circulation, 85(2), 648-658.

Carlson, L. (2012). Knowledge of cervical cancer pathology of high school students in San Carlos, Costa Rica. ACM Field Research in the Environment, Social Sciences \& Humanities, San José, Costa Rica.

Centers for Disease Control and Prevention. (2013). Smoking and tobacco use. Recuperado de http://www.cdc.gov/ Tobacco/

Clark, S. M., \& Nakad, R. (2011). Pharmacotherapeutic management of nicotine dependence in pregnancy. Obstetrics and Gynecology Clinics of North America, 38(2), 297-+. doi: 10.1016/j.ogc.2011.02.017

Dregan, A., Stewart, R., \& Gulliford, M. C. (2013). Cardiovascular risk factors and cognitive decline in adults aged 50 and over: a population-based cohort study. Age and Ageing, 42(3), 338-345. doi: 10.1093/ageing/afs166

Feldman, C., \& Anderson, R. (2013). Cigarette smoking and mechanisms of susceptibility to infections of the respiratory tract and other organ systems. Journal of Infection, 67(3), 169-184. doi: 10.1016/j.jinf.2013.05.004

Gobierno de Costa Rica. (2012). Ley General de Control de Tabaco y sus Efectos Nocivos a la Salud. San José, Costa Rica.

IAFA. (2011). Juventud escolarizada y drogas: encuesta nacional sobre consumo de drogas en población de educación secundaria. San José, Costa Rica: Instituto Sobre Alcoholismo y Farmacodependencia.

IAFA. (2012). Consumo de drogas en Costa Rica: encuesta nacional 2010. San José, Costa Rica: Instituto Sobre Alcoholismo y Farmacodependencia.

INEC: Instituto Nacional de Estadística y Censos (Costa Rica). (2011). Censo Nacional. Recuperado de http://www.inec. go.cr/Web/Home/pagPrincipal.aspx

Metelitsa, A. I., \& Lauzon, G. J. (2010). Tobacco and the skin. Clinics in Dermatology, 28(4), 384-390. doi: 10.1016/j. clindermatol.2010.03.021

Nebot, M., Tomas, Z., Ariza, C., Valmayor, S., Lopez, M. J., \& Juarez, O. (2004). Factors associated with smoking onset: 3-year cohort study of schoolchildren. Archivos De Bronconeumologia, 40(11), 495-501. doi: 10.1016/ s1579-2129(06)60364-9

Prasad, D. S., Kabir, Z., Das, B. C., \& Dash, A. K. (2009). Smoking and cardiovascular health: a review of the epidemiology, pathogenesis, prevention and control of tobacco. Indian Journal of Medical Sciences, 63(11), 520-533. doi: 10.4103/0019-5359.58884

Rahman, M., Mahmood, M., Spurrier, N., Rahman, M., \& Leeder, S. (2011). A systematic review of epidemiological studies on the association between smokeless tobacco use and coronary heart disease. Journal of Public Health and Epidemiology, 3(12), 593-603. doi: 10.5897/JPHE11.175 
Richardson, A., He, J.-P., Curry, L., \& Merikangas, K. (2012). Cigarette smoking and mood disorders in US adolescents: sex-specific associations with symptoms, diagnoses, impairment and health services use. Journal of Psychosomatic Research, 72(4), 269-275. doi: 10.1016/j. jpsychores.2012.01.013

Roth, L. K., \& Taylor, H. S. (2001). Risks of smoking to reproductive health: assessment of women's knowledge. American Journal of Obstetrics and Gynecology, 184(5), 934-939. doi: $10.1067 / \mathrm{mob} .2001 .112103$

Rozen, T. D. (2011). A history of cigarette smoking is associated with the development of cranial autonomic symptoms with migraine headaches. Headache, 51(1), 85-91. doi: 10.1111/j.1526-4610.2010.01707.x

Song, A. V., Morrell, H. E. R., Cornell, J. L., Ramos, M. E., Biehl, M., Kropp, R. Y., \& Halpern-Felsher, B. L. (2009). Perceptions of smoking-related risks and benefits as predictors of adolescent smoking initiation. American Journal of Public Health, 99(3), 487-492. doi: 10.2105/ajph.2008.137679

Steliga, M. A., \& Dresler, C. M. (2011). Epidemiology of lung cancer: smoking, secondhand smoke, and genetics.
Surgical Oncology Clinics of North America, 20(4), 605-+. doi: 10.1016/j.soc.2011.07.003

Surgeon General's Report. (2004). The health consequences of smoking: a report of the Surgeon General. (2004). Atlanta. GA: U.S. Dept. of Health and Human Services, Public Health Service, Office of the Surgeon General.

Talhout, R., Schulz, T., Florek, E., van Benthem, J., Wester, P., \& Opperhuizen, A. (2011). Hazardous compounds in tobacco smoke. International Journal of Environmental Research and Public Health, 8(2), 613-628. doi: 10.3390/ ijerph8020613

Warren, C. W., Riley, L., Asma, S., Eriksen, M. P., Green, L., Blanton, C.,... Yach, D. (2000). Tobacco use by youth: a surveillance report from the Global Youth Tobacco Survey project. Bulletin of the World Health Organization, 78(7), 868-876.

WHO Costa Rica. (2011). NCD Country Profiles: Costa Rica. Retrieved from http://www.who.int/nmh/countries/ cri_en.pdf?ua $=1$

Zhu, K., Moriarty, C., Caplan, L. S., \& Levine, R. S. (2007). Cigarette smoking and primary liver cancer: a population-based case-control study in US men. Cancer Causes \& Control, 18(3), 315-321. doi: 10.1007/s10552-006-0105-8.

Apéndices digitales: Incluye Figuras de la 1 a la 9. Tablas de 2 a la 6 . Apéndice A. 


\section{APÉNDICES}

Apéndice A. Cuestionario.

\section{CUESTIONARIO}

CONOCIMIENTOS Y PERCEPCIONES DEL TABAQUISMO Y SUS EFECTOS DAÑINOS A LA SALUD

El siguiente cuestionario busca evaluar los conocimientos y percepciones de adolescentes sobre el tabaquismo y sus daños a la salud. Es completamente anónimo, y no busca datos personales de los cuales se puede identificar al participante.

El cuestionario consiste en dos partes. La primera contiene todas las preguntas de esta página. Cuando ha terminado esta parte, favor de entregar esta página y pedir la segunda parte, que está en otra página.

\section{PARTE UNO}

Últimos 4 dígitos del teléfono de su casa: Mes y día de nacimiento:

Edad: Año que cursa: Sexo: femenino $\square$ masculino

1. Mencione las enfermedades y complicaciones específicas a la salud que pueden presentar las personas que usan regularmente uno o más productos de tabaco.

2. Estime la importancia de los siguientes factores posibles que puedan influir en la tendencia del tabaquismo adolescente. Puede escribir 1, 2, 3, o"no sé" al lado de cada factor. No hay respuesta correcta ni incorrecta; solo refleja su opinión.

( 1 = no influye, 2 = influye un poco, 3 = influye bastante, "no sé" = no puedo estimar la influencia de este factor)

\section{Factor Estimación de su influencia}

2a. El adolescente desconoce los daños que produce el uso del tabaco a la salud..............

$2 \mathrm{~b}$. El tener hermanos y/o padres que usan productos de tabaco $\ldots \ldots \ldots \ldots \ldots \ldots \ldots \ldots \ldots \ldots$

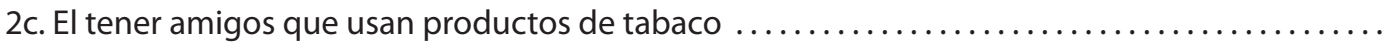

$2 d$. El creer que el fumar es divertido y/o "chiva".

2e. El pasar parte del tiempo libre en fiestas.

2f. otro factor (explique): 


\section{CUESTIONARIO}

CONOCIMIENTOS Y PERCEPCIONES DEL TABAQUISMO Y SUS EFECTOS DAÑINOS A LA SALUD

\section{PARTE DOS}

Últimos 4 dígitos del teléfono de su casa:

Mes y día de nacimiento:

Favor de responder a las siguientes preguntas con detalle en el espacio disponible. Si no sabe como responder, puede solicitar ayuda o simplemente escribir "No sé."

3. Explique el/los efecto(s) que produce el fumar en los pulmones.

4. Explique el/los efecto(s) que produce el fumar en el corazón y las arterías.

5. Explique el/los efecto(s) que produce el fumar a la salud reproductiva femenina y las mujeres embarazadas.

6. ¿En su opinión, cuál sería el daño a la salud más grave de usar los productos de tabaco (no necesariamente tiene que relacionar con los temas de preguntas 3, 4, o 5)? Explique.

¡Muchas gracias por su participación! 
